\title{
Éducation pour la santé en périnatalité : pertinence de l'utilisation du carnet de bord dans l'accompagnement du devenir mère
}

\author{
Perinatal health education: relevance of a logbook in motherhood coaching \\ Marie-Reine BERNARD et Chantal EYMARD \\ Département Sciences de l'éducation, Aix Marseille I- Université de Provence, Unité mixte de recherche « Apprentissage \\ Didactique Éducation Formation », EU3M (École Universitaire de Maïeutique Marseille Méditerranée) Campus Santé \\ Nord, Boulevard Pierre Dramard, 13344 Marseille Cedex 15, France
}

Manuscrit reçu le 25 mai 2009; commentaires éditoriaux formulés aux auteurs le 7 décembre 2009; accepté pour publication le 21 décembre 2010

\author{
Mots clés : \\ Éducation pour \\ la santé ; \\ périnatalité ; \\ carnet de bord ; \\ récit de vie ; \\ réorganisation \\ identitaire maternelle
}

Keywords:

Health education; perinatality; storybook narrative; life story; mother's identity reorganisation
Résumé - Contexte et problématique : Devenir mère occasionne d'importants remaniements physiologiques chargés d'un potentiel déstabilisant et plus rarement, pathologique. Plusieurs études décrivent les effets bénéfiques de l'utilisation du récit de vie dans des actions d'éducation pour la santé visant le soutien psychologique ou thérapeutique. Objectifs : Cette étude questionne la pertinence de l'utilisation du carnet de bord dans une démarche d'éducation pour la santé en post-partum, visant à soutenir les réaménagements identitaires maternels et à prévenir les difficultés qui peuvent leur être corrélées. Méthodes : Une enquête qualitative a été menée auprès de six femmes pendant trois mois. L'entretien non directif a été utilisé pour le recueil des données et la méthode de l'analyse de contenu pour leur traitement. Résultats et discussion : Les résultats ont permis de catégoriser de nombreux thèmes de la réorganisation identitaire issus du corpus théorique. Qu'ils soient communs à plusieurs femmes ou propres à une seule, les thèmes sont articulés de façon singulière. Malgré ses limites, le carnet de bord joue un rôle de médiation relationnelle et facilite le centrage sur le «devenir mère » et ses réaménagements. Le récit de vie qu'il occasionne contribue à la réorganisation identitaire. Il justifie ainsi sa pertinence comme outil dans un dispositif d'éducation pour la santé.

Abstract - Context and problematic: Becoming a mother entails significant physiological changes, which potentially could be destabilizing and sometimes pathological. Several studies describe the benefits of using "life story" in the context of health education as a means for psychological or therapeutic support. Aim: This study examines the appropriateness of using a narrative logbook in a post-partum health education process designed to support identity transformations and prevent correlated difficulties. Methods: A three-month qualitative survey was conducted with six women. The non-directive interview was used for data collection while the "content analysis method" was used for processing. Results and discussion: The results helped identify several issues related to identity reorganization, which stems from the theoretical corpus. Whether the issues are common to several women or individual, they are 
structured in a specific manner. Despite its limitations, the logbook plays the role of a relational mediation and helps to focus on the "becoming a mother" and its associated reorganization. The life story triggered by the logbook contributes to identity reorganization. As a result, it justifies its relevance as a tool in the health education system.

\section{Introduction et problématique}

Devenir mère est une étape physiologique de la vie d'une femme. C'est aussi une expérience qui peut s'effectuer dans la souffrance psychique. Selon les sources, dix à douze pour cent ${ }^{[1]}$, voire quinze pour cent des femmes ${ }^{[2]}$ vivraient une dépression du postpartum, sans avoir nécessairement présenté des facteurs de risques. La dépression en période périnatale apparaît alors comme un problème de santé publique. L'idée de prévention précoce appréhendée dans une perspective de respect du sujet et selon un modèle dit «d'expression » ${ }^{[3]}$ plaide en faveur d'un soutien à la parentalité «naissante » ${ }^{[4]}$, s'inscrivant dans une démarche d'éducation pour la santé.

Plusieurs études réalisées dans le cadre d'une psychothérapie ou d'un soutien psychologique, auprès d'adolescents ${ }^{[5]}$, de personnes âgées ${ }^{[6]}$ ou de femmes atteintes d'un cancer du sein ${ }^{[7]}$ mettent en évidence les effets bénéfiques du récit de vie écrit. Elles rapportent une élévation du sentiment de satisfaction de la prise en charge en cas de pathologie, une augmentation des émotions positives et une diminution des symptômes de dépression dans tous les cas. Une étude qualitative réalisée en alcoologie ${ }^{[8]}$ insiste sur l'importance de la prise en compte de l'histoire de vie dans l'accompagnement thérapeutique. L'histoire de vie est la construction chargée de sens qui résulte du récit de vie destiné à l'Autre ${ }^{[9]}$. Ces résultats ouvrent la voie de l'utilisation du carnet de bord, support du récit que les femmes font de leur vie et de leur vécu, dans un programme d'éducation pour la santé en périnatalité.

L'objectif de la présente étude est de déterminer le potentiel d'utilisation du carnet de bord dans une proposition d'éducation pour la santé en postpartum, visant à soutenir les réaménagements identitaires maternels et à prévenir les difficultés qui peuvent leur être corrélées. Une enquête qualitative a été menée auprès de femmes volontaires ayant tenu un carnet de bord après la naissance de leur enfant. Le but était de retrouver dans leurs récits respectifs les éléments singuliers et significatifs permettant d'appréhender la construction identitaire et d'identifier la place du récit de vie dans cette réorganisation. L'entretien non directif a été retenu comme méthode. Avant de présenter les principaux résultats de l'étude, la discussion et les perspectives d'utilisation du carnet de bord support du récit de vie des femmes en périnatalité, les concepts de santé, d'éducation pour la santé et de réorganisation identitaire maternelle seront précisés.

\section{Cadre conceptuel}

En référence à la charte d'Ottawa (1986) et aux définitions de l'Organisation mondiale de la santé (OMS 1973, 1993, 2003), la santé peut être appréhendée en tant que processus d'adaptation d'un sujet plus ou moins autonome, en fonction de son environnement et des évènements qui constituent sa vie ${ }^{[10]}$. En tant que processus, l'adaptation relève « des compétences personnelles et interpersonnelles, cognitives et physiques qui permettent à des individus de mâ̂triser et de diriger leur existence et d'acquérir la capacité à vivre dans leur environnement et à modifier celui-ci ${ }^{[11]} \gg$. Ces compétences s'inscrivent dans un ensemble plus large de compétences psychosociales : «se connaître soi-même, avoir confiance en soi ; savoir gérer ses émotions et maîtriser son stress; développer un raisonnement créatif et une réflexion critique; développer des compétences en matière de communication et de relations interpersonnelles ; prendre des décisions et résoudre un problème; se fixer des buts à atteindre et faire des choix; s'observer, s'évaluer et se renforcer ${ }^{[12]}$ ». Éminemment subjective, la santé est donc une façon unique et singulière d'exister ${ }^{[10]}$ et « un mode 
de présence au monde ${ }^{[13]} »$, c'est-à-dire à l'Autre, ce qui ne permet pas d'éluder la question du sens de ce vivre. Elle fait référence à la notion de qualité de vie définie par l'OMS (1993) comme «la perception qu'a un individu de sa place dans l'existence, dans le contexte de la culture et du système de valeurs dans lesquels il vit, en relation avec ses objectifs, ses attentes, ses normes et ses inquiétudes. C'est un concept très large, influencé de manière complexe par la santé physique du sujet, son état psychologique, son niveau d'indépendance, ses relations sociales ainsi que sa relation aux éléments essentiels de son environnement ${ }^{[14]} »$. L'éducation pour la santé peut alors être appréhendée comme une démarche qui se propose d'accompagner le sujet dans ce processus dont les dimensions biologique, psychologique, sociale et culturelle sont indissociables ${ }^{[15]}$.

La notion d'éducation est polysémique. Elle prend tantôt le sens d'instruction et d'acquisition de savoirs, tantôt celui de développement de compétences ou de comportements d'adaptation, mais aussi de connaissance de soi, de développement de l'autonomie ou de la socialisation des personnes ${ }^{[16]}$. L'éducation ne peut en conséquence se réduire à de l'information car elle vise l'appropriation de savoirs ou de savoir-faire, se propose d'accompagner le sujet dans un processus de changement ${ }^{[10]}$, en l'invitant «à construire son devenir » ${ }^{[16]}$, à développer « la capacité à faire des choix, à décider par soi-même et à exister en tant que citoyen autonome dans un environnement sociétal » ${ }^{[16]}$.

En périnatalité, l'éducation pour la santé « comprend toutes les activités visant intentionnellement l'accroissement des connaissances en matière de santé et le développement d'aptitudes influençant positivement la santé ${ }^{[15]}$ des femmes. Au sens promotion de la santé, elle s'attache à promouvoir les «modes de vie aussi bien que l'amélioration des conditions de vie, des facteurs sociaux, économiques et environnementaux qui déterminent la santé » ${ }^{[17]}$. C'est une démarche globale centrée sur les femmes qui fait appel au concept d'empowerment $^{[15,18]}$. Il s'agit d'une approche humaniste qui s'adresse aux femmes engagées dans une relation de soins basée sur leur participation active $^{[15]}$ et n'échappe pas à la question éthique ${ }^{[19]}$. Le but est d'augmenter l'autonomie des femmes, composante de leur santé, en facilitant l'acquisition ou le maintien des compétences dont elles ont besoin pour gérer et s'adapter aux changements inhérents à la maternité ${ }^{[18]}$. Les femmes occupent une place de sujets des soins qui leur sont proposés et bénéficient d'un suivi régulier et personnalisé dans un climat d'écoute active, sur toute la période périnatale ${ }^{[15]}$. Des réponses qui leur sont apportées, des choix qui sont faits par les femmes, dépend en partie la trajectoire de leur vie mais il ne peut leur être reproché de ne pas avoir développé les compétences attendues.

Les théories du champ de la psychanalyse indiquent clairement que la naissance d'un enfant provoque, chez la femme qui devient ou redevient mère, une véritable crise identitaire ${ }^{[20-23]}$. Ce bouleversement appartient aux processus physiologiques constitutifs du «devenir mère ». Des disciplines comme la philosophie, la sociologie, la psychologie ou les sciences de l'éducation permettent d'appréhender la complexité du concept d'identité maternelle et de sa réorganisation systémique, à la fois mouvement interne et dynamique externe, dépendante des interactions avec autrui. Les processus psycho-affectifs, cognitifs ou identitaires, ne sont pas en concurrence au cours de leur évolution mais peuvent interagir entre eux de façon positive ou négative. Ainsi, des difficultés d'ordre identitaire peuvent perturber l'ensemble des processus, retentir sur la santé psychique de la mère, sur la relation mère-enfant et sur le développement de l'enfant. L'identité narrative ${ }^{[24-26]}$ générée par le récit que l'on fait de soi est une dimension de l'identité qui contribue à son unité car «c'est dans et par le langage que l'homme se constitue, se structure comme sujet » ${ }^{[27]}$. Par ailleurs, le récit de vie est l'occasion d'élaborer « une nouvelle réalité interactive » ${ }^{[28]}$ qui favorise la socialisation, ce qui n'est pas sans intérêt dans une perspective d'accompagnement.

Les remaniements identitaires féminins dans la période post-natale prennent place dans les 
nombreux changements qui viennent émailler le « devenir mère » et influencer la santé de la femme. Leur prise en compte dans une démarche d'éducation pour la santé en périnatalité semble incontournable. L'utilisation du carnet de bord consignant le récit de vie des mères comme outil de cette démarche est encourageante. Nous nous proposons donc d'en étudier la pertinence et les perspectives d'utilisation dans des actions d'éducation pour la santé menées par les sages-femmes.

\section{Participantes et méthodes}

La recherche s'est déroulée dans une petite maternité française (moins de 200 naissances par an) ne recevant que des femmes dont le pronostic d'accouchement est évalué à bas risque, entre octobre 2007 et mars 2008. Les participantes à l'enquête ont été sélectionnées sur la base du volontariat, le seul critère d'inclusion étant d'avoir accouché par voie basse. Initialement, quatorze femmes ont été rencontrées entre le jour de l'accouchement et trois jours après celui-ci. La première rencontre a eu lieu dans le cadre institutionnel où le chercheur principal exerçait ponctuellement comme sage-femme. Le travail de recherche a été clairement défini et situé hors du cadre hospitalier et sans rapport direct avec le rôle professionnel du chercheur dans le service. Quatre ont refusé d'emblée la proposition au motif qu'elles n'étaient pas intéressées, n'aimaient pas écrire ou n'auraient pas le temps de le faire. Dix ont accepté verbalement de tenir un carnet de bord et de s'entretenir une fois par mois avec le chercheur. Au bout d'un mois, trois ont manifesté leur souhait de se retirer du dispositif car elles n'avaient pas écrit. Une autre a insisté pour réaliser les entretiens sans tenir de carnet de bord; elle a donc été exclue de l'étude. La recherche a finalement concerné six femmes (tableau I).

L'écriture a commencé la semaine de la naissance et s'est poursuivie pendant trois mois. Un rythme d'écriture d'au moins deux fois par semaine a été proposé. Dans le carnet de bord, les mères ont été invitées à consigner leur vécu de la relation qu'elles construisaient avec leur enfant, leur ressenti à l'égard des changements qui s'opéraient dans la famille, les difficultés qu'elles affrontaient et/ou satisfactions qu'elles vivaient. Il a été précisé que le chercheur n'effectuerait aucun contrôle du carnet de bord et que celui-ci resterait leur propriété.

Pendant cette période, des entretiens non directifs ont eu lieu une fois par mois. Ils se sont déroulés au domicile des femmes et ont été enregistrés intégralement. Le choix des contenus thématiques de ces rencontres, guidé par leur carnet de bord, a été laissé aux femmes. Un total de quatorze entretiens (tableau I) correspondant à des périodes d'écriture des six participantes ont été retranscrits et traités selon la méthode de l'analyse de contenus ${ }^{[29]}$. La lecture des interviews retranscrites et regroupées pour chaque participante a permis de dégager des thèmes en coïncidence avec le corpus théorique. Les interviews ont été relues de façon indépendante par le second chercheur afin de s'assurer de la concordance de ces thèmes émergents.

\section{Principaux résultats}

Les thèmes de la construction identitaire retrouvés dans les verbatim des mères sont regroupés dans les tableaux II et III. L'émergence d'un nombre élevé de thèmes a nécessité un important travail de catégorisation qui a mis en évidence la singularité du réagencement des éléments constitutifs de l'identité. La présence d'éléments communs à plusieurs femmes ne conduit pas à une réorganisation similaire car la relation entre l'identité et les différents éléments qui participent à cette réorganisation n'est pas de type linéaire ${ }^{[1]}$. La multiplicité des influences et la complexité des réaménagements permet d'appréhender la fragilité potentielle de la réorganisation identitaire maternelle, offrant simultanément aux professionnels de la périnatalité un grand nombre d'opportunités d'apporter aide et soutien dans une démarche d'éducation pour la santé.

\section{Relation mère enfant}

L'identification de la femme à sa mère ${ }^{[30]}$ est signalée quand elle pose problème, soit du fait de 
Tableau I. Présentation des femmes, chronogramme des entretiens et durée d'enregistrement de ceux qui correspondent à une période d'écriture et ont été analysés.

\begin{tabular}{|c|c|c|c|c|c|c|c|c|}
\hline \multicolumn{3}{|c|}{ Entretiens analysés : E.1, E.2, E.3; } & \multicolumn{6}{|c|}{ Entretiens non analysés : (1), (2), (3) } \\
\hline & & $\begin{array}{l}2007 \\
\text { Fin nov. }\end{array}$ & $\begin{array}{l}\text { Début } \\
\text { déc. }\end{array}$ & $\begin{array}{l}2008 \\
\text { Début } \\
\text { janvier }\end{array}$ & $\begin{array}{l}\text { Début } \\
\text { février }\end{array}$ & $\begin{array}{l}\text { Début } \\
\text { avril }\end{array}$ & $\begin{array}{l}\text { Début } \\
\text { mai }\end{array}$ & $\begin{array}{l}\text { Début } \\
\text { juin }\end{array}$ \\
\hline $\begin{array}{l}\text { Amélie } \\
\text { Primigeste, primipare. } \\
\text { Naissance d'Alice par ventouse. } \\
\text { Allaitement maternel. }\end{array}$ & 27 ans & $\begin{array}{l}\text { E. } 1 \\
25 \text { min }\end{array}$ & & $\begin{array}{l}\text { E. } 2 \\
30 \text { min }\end{array}$ & $\begin{array}{l}\text { E. } 3 \\
30 \text { min }\end{array}$ & & & \\
\hline $\begin{array}{l}\text { Carole } \\
\text { Secondigeste, secondipare. } \\
\text { Maman de Marine, } 2 \text { ans. } \\
\text { Naissance eutocique d'Arthur. } \\
\text { Allaitement maternel. }\end{array}$ & 35 ans & $\begin{array}{l}\text { E.1 } \\
30 \text { min }\end{array}$ & & $\begin{array}{l}\text { E. } 2 \\
35 \text { min }\end{array}$ & $\begin{array}{l}\text { E. } 3 \\
40 \text { min }\end{array}$ & & & \\
\hline $\begin{array}{l}\text { Danièle } \\
\text { Primigeste, primipare. } \\
\text { Naissance eutocique de Sam. } \\
\text { Allaitement maternel, fortes douleu } \\
\text { le } 1^{\text {er }} \text { mois. }\end{array}$ & 30 ans & & $(1)$ & $\begin{array}{l}\text { E. } 2 \\
40 \text { min }\end{array}$ & $\begin{array}{l}\text { E. } 3 \\
50 \text { min }\end{array}$ & & & \\
\hline $\begin{array}{l}\text { Elsa } \\
\text { Primigeste, primipare. } \\
\text { Naissance eutocique de Jeanne. } \\
\text { Allaitement maternel très difficile } \\
\text { puis arrêt. }\end{array}$ & 39 ans & $\begin{array}{l}\text { E.1 } \\
35 \text { min }\end{array}$ & & $(2)$ & (3) & & & \\
\hline $\begin{array}{l}\text { Joséphine } \\
\text { Primigeste, primipare. } \\
\text { Naissance eutocique de Salomé. } \\
\text { Allaitement maternel. }\end{array}$ & 28 ans & & & & & $\begin{array}{l}\text { E. } 1 \\
80 \text { min }\end{array}$ & $\begin{array}{l}\text { E. } 2 \\
65 \text { min }\end{array}$ & $(3)$ \\
\hline $\begin{array}{l}\text { Sarah } \\
\text { Secondigeste, secondipare. } \\
\text { Maman de Maï, } 3 \text { ans. } \\
\text { Naissance eutocique de Galaad. } \\
\text { Allaitement maternel. }\end{array}$ & 36 ans & $\begin{array}{l}\text { E. } 1 \\
30 \mathrm{~min}\end{array}$ & & $\begin{array}{l}\text { E. } 2 \\
30 \text { min }\end{array}$ & $\begin{array}{l}\text { E.3 } \\
50 \text { min }\end{array}$ & & & \\
\hline
\end{tabular}

l'absence de la mère - "mon rôle de mère, je savais pas trop ce que c'était non plus, qu'est-ce que c'était d'être mère est-ce que?... » (Amélie) -, soit en raison d'une identification négative déclarée «j'avais pas spécialement envie de faire la même chose » (Carole).

La dette de vie à l'égard de sa propre mère est indispensable pour accéder à la maternité ${ }^{[30]}$; même si l'on ne s'identifie pas à elle, on peut néanmoins lui reconnaître un certain mérite : «déjà qu'elle nous voulait pas, en plus elle s'est retrouvée entre quatre murs avec deux gamins... (petit rire) c'est pas facile» (Carole).

La symbiose mère/enfant est une phase normale de la relation, qui fait suite à la grossesse et s'inscrit dans la «préoccupation maternelle précoce » décrite par Winnicott ${ }^{[31]}$. C'est au cours de cette période que se produit une identification croisée de l'un vers l'autre, qui permet à l'enfant de se sentir exister - «... i' nous raconte sa nuit. Enfin, c'est 
Tableau II. Principaux thèmes émergents de la réorganisation identitaire de chaque femme, issus du corpus théorique (I).

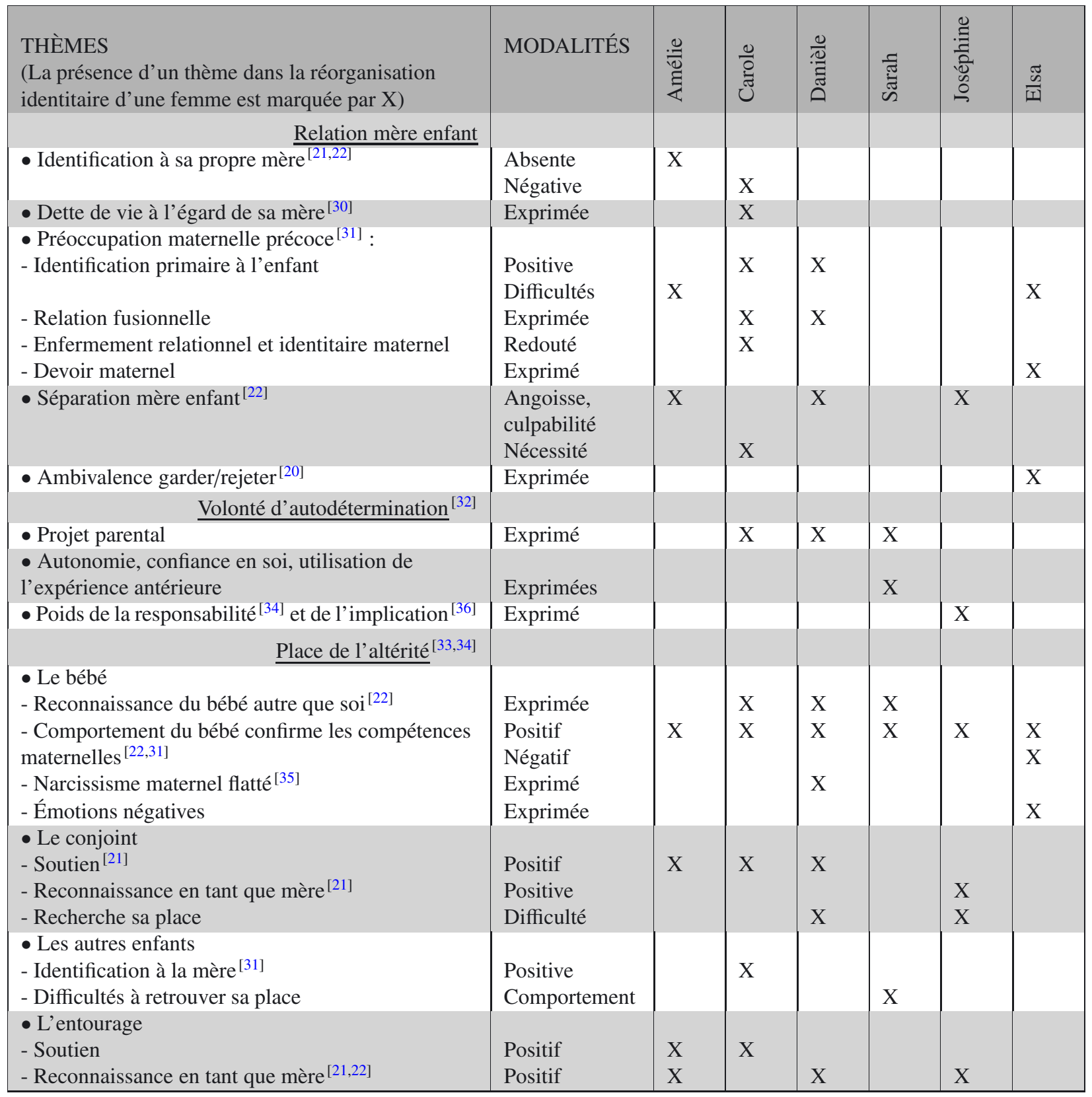

un bébé qui communique beaucoup »- et c'est à la mère de comprendre les besoins de son bébé j'ai, j'ai pas de pleurs incompréhensibles [...] Ça paraît évident...»(Danièle). En revanche, quand l'identification primaire ${ }^{[31]}$ à son bébé est perturbée, la mère a le sentiment de ne pas satisfaire ses besoins - il a «toujours faim » ou n' «a pas pris de poids » (Elsa) - et elle se vit insuffisante, voire incapable. La relation en pâtit : «ça me gâchait la relation »; s'occuper de son enfant devient un devoir - «on a signé, on va continuer » - et plus rarement une satisfaction - «j'ai réussi à la rendre bien. 
Tableau III. Principaux thèmes émergents de la réorganisation identitaire de chaque femme, issus du corpus théorique (II).

\begin{tabular}{|c|c|c|c|c|c|c|c|}
\hline $\begin{array}{l}\text { THÈMES } \\
\text { (La présence d'un thème dans la réorganisation } \\
\text { identitaire d'une femme est marquée par X) }\end{array}$ & MODALITÉS & 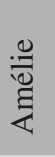 & 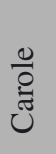 & 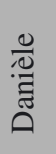 & 胥 & 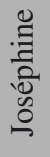 & $\frac{\pi}{\infty}$ \\
\hline \multicolumn{8}{|l|}{$\underline{\text { Influences culturelles et de la société }}{ }^{[25]}$} \\
\hline - Mode et stéréotypes véhiculés par les médias & Exprimés & & & & & $X$ & \\
\hline \multicolumn{8}{|l|}{ Réactions face aux changements et à la perception de } \\
\hline \multicolumn{8}{|l|}{ l'incertitude qu'ils occasionnent } \\
\hline - Déni du changement & Exprimé & $\mathrm{X}$ & & & & $\mathrm{X}$ & \\
\hline - Ambiguïté du rapport au temps ${ }^{[34]}$ & Exprimée & $\mathrm{X}$ & & & & $\mathrm{X}$ & \\
\hline - Projections dans le long terme & Exprimées & $\mathrm{X}$ & $X$ & & $\mathrm{X}$ & & \\
\hline - Importance de la maîtrise et intégrité du corps & Exprimée & & & & & $\mathrm{X}$ & \\
\hline \multicolumn{8}{|l|}{ Place et apports de l'écriture } \\
\hline - Trace intergénérationnelle, souvenir pour soi & Exprimés & $\mathrm{X}$ & $\mathrm{X}$ & & $\mathrm{X}$ & $\mathrm{X}$ & $\mathrm{X}$ \\
\hline - Aide à la prise de conscience ${ }^{[28]}$ & Exprimée & $\mathrm{X}$ & $\mathrm{X}$ & $\mathrm{X}$ & $\mathrm{X}$ & $\mathrm{X}$ & \\
\hline - Aide à la prise de distance ${ }^{[28]}$ & Exprimée & $X$ & $\mathrm{X}$ & $\mathrm{X}$ & $\mathrm{X}$ & $\mathrm{X}$ & \\
\hline - Attribution de sens aux évènements ${ }^{[28]}$ & Exprimée & $X$ & & $\mathrm{X}$ & $\mathrm{X}$ & $\mathrm{X}$ & \\
\hline - Ouverture, communication avec le conjoint ${ }^{[34,38]}$ & Exprimées & $X$ & & $\mathrm{X}$ & & & \\
\hline $\begin{array}{l}\text { - Réflexion, questionnement sur soi, } \\
\text { compréhension de soi }{ }^{[24]}\end{array}$ & Exprimés & $\mathrm{X}$ & $\mathrm{X}$ & $\mathrm{X}$ & $\mathrm{X}$ & & \\
\hline - Amélioration de l'autonomie & Exprimée & & & $\mathrm{X}$ & $\mathrm{X}$ & & \\
\hline - Affirmation de sa singularité ${ }^{[28]}$ & Exprimée & & $\mathrm{X}$ & $\mathrm{X}$ & & $\mathrm{X}$ & \\
\hline
\end{tabular}

Ça fait plaisir » (Elsa). De la difficulté d'identification primaire peut aussi résulter une difficulté de reconnaissance de son enfant quand, par exemple, il n'est pas orphelin comme l'a été sa mère - «et, me dire qu'elle a une maman aussi » (Amélie). L’hésitation retentit sur la réorganisation identitaire de la mère et la construction des liens. La proximité privilégiée et rassurante de la phase fusionnelle précoce est nécessaire et apaisante pour la mère et l'enfant - «quand on l'a dans les bras il apaise en même temps qu'il se fait apaiser, c'est agréable » (Carole). Néanmoins, elle doit prendre fin.

Les mères évoquent souvent la séparation avec appréhension - «le laisser ça va être dur je crois [...] toutes mes angoisses, tout mon complexe à moi, d'abandon » (Danièle) -, parfois avec culpabilité - «est-ce que je la laisse pas trop tôt? [...] c'est un peu paradoxal, je me sens pas vraiment maman et en même temps je culpabilise de la laisser et...» (Amélie) - ou bien avec soulagement provisoire - "ouf enfin on a un peu de temps pour soi, ah oui mais finalement ouh elle me manque » (Joséphine). Pour d'autres, c'est une évidence - «il va falloir le lâcher » (Sarah) -, voire une nécessité pour échapper à l'enfermement relationnel pathologique - «c'est pour ça qu'on hésite pas à les confier»(Carole). La mise en tension entre relier/délier n'est jamais étrangère à l'histoire personnelle des femmes pendant leur propre enfance.

L'ambivalence entre garder et rejeter, entre amour et haine ${ }^{[20]}$ est caractéristique des réaménagements psychiques, émotionnels et identitaires liés à la naissance, elle conduit à des sentiments contradictoires de «dualité » qui oscillent entre «on 
l'adore », un «mouvement de recul» ou une envie réprimée de «pas si on l'avait pas» (Elsa), qui sont rarement aussi clairement explicités.

\section{Volonté d'autodétermination}

Si l'on se réfère à la théorie de l'autodétermination ${ }^{[32]}$, être autonome, c'est décider de son action et réaliser cette action. Dans cette perspective, autonomie et dépendance ne sont pas incompatibles puisqu'une dépendance choisie n'entrave pas l'autonomie. Les études faites à ce sujet démontrent que les sentiments de bien-être et l'autonomie sont conservés quand les personnes déterminent leurs choix. C'est aussi ce qu'expriment les femmes - «t'as choisi cette relation là, t'as choisi de pas reprendre le boulot, t'as choisi l'allaitement, donc tu as choisi de rester dans cette relation là avec ton bébé » (Danièle); «si j'ai choisi d'avoir des enfants, c'est aussi parce que je me sens prête à ça» (Carole). Gagner en autonomie - «Oui, puis moi, j'ai beaucoup d' mal à faire des choix »- est parfois un long apprentissage qui, d'un enfant à l'autre, évolue favorablement : «je dis, oui oui pourquoi pas, et puis voilà. Alors que pour le premier... »(Sarah).

\section{Place de l'altérité}

L'intersubjectivité est au cœur des relations humaines et la définition du sujet, même si elle n'est que provisoire, se fait par rapport à l'Autre ${ }^{[33,34]}$, qu'il s'agisse de l'entourage, du conjoint ou des enfants.

Très tôt l'enfant s'affirme comme Autre - «...et il est vraiment lui... il est incroyable » (Danièle), «c'est vraiment des petits êtres... " (Carole). Dans ses interactions avec sa mère qui évoluent avec l'âge ${ }^{[35]}$, il la contraint au changement et influence la réorganisation de son identité - «c'est quelqu'un qui occupe de la place dans la maison [...] lui pas encore, pour le moment c'est le bébé qu'on pose quelqu' part... »(Carole) -. Parfois, l'enfant renvoie à sa mère, une image de mère incompétente - «on a l'impression qu'on va pas y arriver [... ] et que nous on sait pas faire » (Elsa) -, ou au contraire flatte son narcissisme ${ }^{[22]}$ - «tout le monde l'a trouvé magnifique! »(Danièle). Selon le cas, les répercutions sur les émotions, le psychisme et le sentiment identitaire maternels risquent de présenter des oscillations contraires.

Les enfants aînés ne sont pas indifférents à la naissance d'un autre enfant. Leurs réactions produisent parfois chez leur mère de la compassion - «je trouve ça dur, pour elle, [...] et que voilà des fois c'est comme si elle disait : je suis en train de faire le deuil d'être enfant unique » (Sarah) - ou, d'autres fois, de l'incompréhension ou du questionnement. La fille de Carole s'engage dans un processus d'apprentissage du maternage par imitation, avec des poupées. Ce comportement interroge la mère - «je me suis demandée si moi, j'avais fait pareil ou pas... » (Carole) - avec d'autant plus de force qu'elle refuse de s'identifier à sa propre mère.

Dans nos sociétés modernes, aux groupes familiaux réduits, le père est le principal soutien de la femme qui devient mère, tandis qu'il négocie ses propres réaménagements identitaires, cognitifs et psychiques et sa nouvelle place dans le couple. Il arrive que la rencontre des altérités ${ }^{[24]}$ soit responsable d'altération ${ }^{[36]}$ de soi aussi perturbante pour la mère que pour le père - «on bataille un peu contre l'autre mais on bataille aussi contre soi» (Danièle) - et qu'à la recherche de reconnaissance mutuelle les tensions s'exacerbent et retentissent sur le devenir parents - «je peux pas lui demander d'être une mère quoi » (Danièle); " j'ai mis qu'on était pas toujours d'accord, [...] que j'essayais de faire des efforts [...] mais que j'avais beaucoup de ressenti $\gg$ (Joséphine).

Les réactions de l'entourage familial peuvent contribuer à renforcer l'estime de soi des mères - «ça me réconforte aussi le fait de me dire que c'est moi qui peut la calmer » (Amélie); «oui voilà, ça me conforte quand j' vois dans le regard des amis, de la famille, qui est assez. . pfou j'allais dire assez admiratif » (Danièle). Au contraire, si les femmes se sentent obligées de se justifier, d'expliquer les raisons de leur lassitude à «des gens qui comprennent 
pas forcément » ou «qui ne savent pas encore ce que c'est et... » (Amélie), les doutes ou la culpabilité les envahissent.

\section{Influences culturelles et sociétales}

Le poids des représentations culturelles et sociétales modifie nos comportements ${ }^{[25]}$. Les rôles parentaux traditionnellement définis, en dépit des remises en cause dont ils ont fait l'objet ces dernières décennies, n'ont pas totalement disparu de nos représentations. À l'heure de l'installation dans leur nouveau rôle maternel, des femmes en subissent encore les influences - «Les papas, c'est beaucoup le jeu et l'amusement. [...] Moi, le rôle de maman, c'est répondre à des besoins, des attentes pas que! pour en profiter quoi » (Joséphine). La vulgarisation de certaines théories ne laisse pas les femmes indifférentes. Qu'il s'agisse d'idées «modernes» (par exemple celle de l'attachement avec notamment les effets de l'ocytocine et du peau à peau) - «on dit que c'est important, mais je pense que c'est même. . . la chose la plus importante » (Joséphine) ou d'autres fort anciennes tel que l'instinct maternel - «on a beau dire y'a pas d'instinct maternel, je pense que quelque part y'a... y'a quand même un truc quoi $\gg$ (Joséphine).

Réactions face aux changements et à la perception de l'incertitude qu'ils occasionnent

Les changements provoquent parfois une perception accélérée du temps ${ }^{[34]}$ - «faut s'adapter rapidement en fait » (Amélie); "ça va vraiment très vite » (Sarah). Ils peuvent aussi être à l'origine de projections anticipatrices du futur qui permettent de relativiser les incertitudes du présent - «J'appréhende oui... ben l'av'nir. L'av'nir dans ma relation avec eux, ce qu'i' peuvent... devenir euh ... [...] y'a des enfants qui un jour tournent mal» (Sarah) ou de déni de leur existence - «...c'est l'évolution, c'est... la même chose [...] et puis ça a pas vraiment évolué et donc oui, au niveau de la nouveauté y'en a pas vraiment» (Amélie); «de voir que les choses elles changent pas... enfin elles évoluent pas forcément. Enfin je veux dire ça évolue, mais c'est pas spécialement différent de ... »(Joséphine). L'identité des femmes est atteinte dans la dimension du maintien de soi durable ${ }^{[34]}$ pour laquelle les remaniements sont toujours perturbants. L'illusion d'un retour au même interroge - «Est-ce que je vais retrouver ma vie, entre guillemets, d'avant?» (Amélie) - ou égare dans le temps - «comme c'est passé tellement vite et que j'ai l'impression que mon accouchement c'était y'a deux semaines » (Amélie). Pour une sportive, elle peut aussi prendre des allures de défi physique - «non j'ai envie de redevenir... enfin, peut-être pas moi d'avant, si, moi d'avant physiquement quand même» (Joséphine).

\section{Place de l'écriture et apports du carnet de bord}

L'absence de mémoire intergénérationnelle motive l'intérêt manifesté par certaines femmes pour l'expérience de récit de vie - «moi j'ai aucune trace de mon enfance » (Carole); "Quand c'est pas écrit i' faut chercher dans nos souvenirs, on se dit mais on est pas vraiment sûr...» (Amélie). Pour toutes les femmes, à l'exception de Danièle, le carnet de bord consigne des récits qui sont ou seront à la fois souvenirs - «C'est. . . une aide au souvenir c'est important...» (Amélie) - et des témoignages - «j'ai écrit ce que je ressentais [...] ce que je pouvais mettre comme sentiments à ce que j'ai vécu » (Sarah) -, même si la trace semble faire l'objet d'une grande incertitude - «peut-être plus pour avoir...quelques souvenirs, on va dire, peut-être... » (Elsa). Le carnet de bord est tantôt le moyen de lutter contre l'oubli - «parce qu'on perd après... le fil de ce qui s'est passé »(Carole) -, tantôt celui de transmettre à l'enfant une histoire dont il ne se souviendra pas - «c'est plus... pour eux [...] pour qu'ils sachent » (Carole); « y'a plein de choses par rapport à Maï que je regrette de pas avoir écrites ou notées » (Sarah).

L'écriture est signalée comme une occasion de réflexion ${ }^{[27]}$ - «amène une réflexion [... ] ça m'aide à me poser des questions, à réfléchir, et euh voilà... voilà, à poser les choses en fait. [...] ça donne 
du sens » (Sarah); " j’y ai réfléchi après, donc en notant, d'où l'intérêt de noter » (Joséphine) et de questionnement - «je me serais pas posée autant de questions sinon...»(Carole). Pour cinq femmes, elle facilite la prise de distance $-\ll$ on a trop l'nez dedans » (Danièle) - et stimule la prise de conscience ${ }^{[28]}$ - «c'est en écrivant que j'en ai pris vraiment conscience » (Danièle); «le fait d'écrire on se rend compte de ce qu'on a ressenti » (Amélie). Elle suscite un mouvement psychique et favorise ainsi une meilleure compréhension de soi et des autres ${ }^{[24]}$ - «c'est en écrivant que j' me suis rendue compte que... » (Danièle) - et une relativisation des faits. L'écriture est aussi une ouverture vers l'Autre, un moyen de rétablir la communication - « si j'arrive pas à lui dire au moins je pourrai lui faire lire » (Danièle) -, de se faire reconnaître par l'Autre ${ }^{[34,38]}$.

\section{Discussion}

Les résultats issus d'un faible effectif de femmes ayant accouché dans un même établissement restent limités au contexte de cette maternité et ne doivent être généralisés qu'avec précaution, bien que coïncidant largement avec la théorie en ce qui concerne les principaux thèmes de la réorganisation identitaire.

Sur quatorze femmes sollicitées, six ont tenu un carnet de bord. Sur les six, seulement la moitié (trois) a mené l'expérience pendant trois mois. Ces chiffres mettent en évidence la difficulté que représente l'écriture en terme de disponibilité intellectuelle, mentale et de temps. L'absence de sa maitrise est un frein à prendre en compte mais l'écriture peut être remplacée par le dessin, capable de la même manière de générer des échanges riches et porteurs de sens. Ainsi, le caractère discriminatoire de l'écriture ne remet pas en cause l'intérêt de l'utilisation du carnet de bord car il peut être le support d'un autre mode d'expression dans une démarche préventive.

À l'évidence, les femmes qui conduisent l'expérience de narrativité pendant trois mois savent se saisir de l'occasion d'accompagnement et de questionnement que celle-ci leur offre. Elles sont sensibles à une approche d'éducation pour la santé qui ne se réduit pas à de l'information ou à une transmission de bonnes pratiques. Elles sont sans doute dotées d'une certaine prédisposition pour la réflexion et ont conscience que devenir mère n'est pas seulement une question biologique. Elles prennent le risque de poursuivre un processus de développement de la connaissance de soi, objectif de toute démarche d'éducation pour la santé ${ }^{[16]}$, qui alimentera le processus d'adaptation qui fait suite à la naissance d'un enfant. Elles font du carnet de bord l'intermédiaire de la relation qu'elles tissent avec le chercheur professionnel de santé. Bien présent lors des entretiens, il symbolise le respect mutuel de l'accord et explicite la confiance réciproque. Il se révèle un outil de médiation pertinent dans une relation d'accompagnement qui remet en question la relation soignantsoigné basée sur la détention d'un savoir. Le cas d'Elsa suggère néanmoins que certaines difficultés du post-partum ou du passé (Elsa a déjà utilisé le carnet de bord) peuvent faire obstacle à l'utilisation du récit de vie, à moins qu'Elsa n'ait pas trouvé dans le chercheur un soutien suffisamment bon pour poursuivre l'expérience. Ainsi, l'efficacité du carnet de bord en terme de prévention semble liée à la qualité des interactions femme-sage-femme, celle-ci dépendant de la capacité de la femme à s'approprier l'outil et de la capacité d'écoute et de sécurisation émanant de la sage-femme. S'il apparaît clairement que cet outil ne peut faire l'économie de la rencontre, c'est qu'il est particulièrement adapté à un modèle d'éducation pour la santé basé sur le partenariat, invitant les femmes à occuper une place de sujet porteur d'un savoir expérientiel, d'une histoire et d'un système de valeurs. Ce modèle «privilégie l'analyse des situations implicantes » ${ }^{[16]}$ afin de favoriser la prise de conscience de sa réalité, de développer la connaissance et l'estime de soi et d'accroître des compétences qui permettent de faire des choix et d'élaborer des projets.

Se questionner sur le rôle de l'identité professionnelle du chercheur semble ici légitime. Il accueille les paroles prononcées avec bienveillance et empathie, sans pouvoir faire totalement abstraction de sa propre subjectivité, de son implication, 
au demeurant directement liées à la nature de son écoute. Ainsi de brefs épisodes où la sage-femme éclipse transitoirement le chercheur sont sans doute repérables. Un exemple au cours du deuxième entretien avec Amélie est isolé. Amélie est en proie à une véritable crise de doutes au sujet de la quantité de lait qu'elle produit; elle semble déstabilisée. La sage-femme prend la parole quelques minutes pour démentir ses incertitudes. L'intervention influe probablement sur le cours des propos d'Amélie. La réassurance relance la réflexion d'Amélie qui semblait se bloquer : "j'ai l'impression qu'i' y'a pas assez non plus avec les deux, donc... ». Après un court silence, elle rapporte des impressions positives qu'elle n'avait pas su utiliser pour diminuer ses angoisses, probablement faute d'en avoir pris conscience. Ce qu'elle dit, elle ne l'aurait peut-être pas dit sans l'intervention qui lui redonne confiance, parce que les émotions négatives neutralisent tout autre ressenti de l'expérience vécue. Dans le cadre de la recherche, cet épisode représente un biais. Dans celui d'une démarche éducationnelle préventive, il rend possible un remuement psychique et la révision d'un écrit négatif ${ }^{[38]}$. Les interactions et échanges langagiers, qui se développent autour des expériences consignées dans le carnet de bord, permettent de mettre à distance les affects envahissants, de reconsidérer les doutes exprimés et de modifier les interprétations des évènements. La présence sécurisante de la sage-femme facilite l'évolution, tandis que le carnet de bord conserve la trace des difficultés rencontrées. L'évaluation du chemin parcouru est possible a posteriori. Amélie y trouve alors du réconfort - «Mais quand on relit après dans le détail, ah ben non c'est vrai que... y'a eu cette phase là, c'était pas facile »- en développant une aptitude à relativiser les faits. Son comportement s'en trouve modifié. La valeur éducative de l'analyse métacognitive, réalisée par les personnes et relative à leur propre vécu factuel et relationnel lors de changements entraînant de profonds remaniements, a été rapportée par la théorie socio-cognitive ${ }^{[16]}$.

Puisque, dans une perspective éducative et préventive, nous cherchions à comprendre comment les différents paramètres constitutifs de l'identité, au nombre desquels figurent la narrativité, font l'objet de réaménagements singuliers, l'analyse de contenu des entretiens se justifiait. Elle a en effet permis leur repérage.

Concernant la méthode, les entretiens basés sur le contenu des carnets de bord permettent une confrontation des femmes au récit qu'elles ont écrit. En l'explicitant face au chercheur, elles tentent d'en agencer les éléments pour atteindre une représentation de soi conforme à l'image qu'elles ont d'ellesmêmes ou qu'elles veulent donner ${ }^{[25,38]}$. Le récit oral qui succède au récit écrit donne lieu à une nouvelle interprétation, qui est la première étape de l'analyse pour la recherche, mais aussi une opportunité d'appropriation de son histoire pour la femme. Cette re-présentation de soi et de l'autre, en jouant le rôle d'organisateur psychique ${ }^{[26,39]}$, est à l'origine de l'identité ${ }^{[38]}$ dont les principaux thèmes constitutifs ont été isolés dans les récits des femmes. Ce choix s'avère donc pertinent car il facilite le centrage sur le «devenir mère » et les réaménagements qu'il occasionne, en même temps qu'il contribue à ces réaménagements. L'utilisation du procédé dans un dispositif d'éducation pour la santé visant le soutien des mères dans leur accession à la parentalité semble pour ces mêmes raisons tout aussi pertinente car l'expérience de narrativité écrite menée par les femmes, en prenant place dans le réaménagement de leur identité, devient un facteur de santé et de développement individuel qui accorde une place à leur subjectivité et à leur autonomie. Le carnet de bord confirme ici sa force comme outil d'une démarche éducative en santé conçue comme accompagnement d'un projet parental des femmes et des couples.

\section{Conclusion et perspectives}

L'usage du récit de vie consigné dans un carnet de bord, présenté dans cette étude, semble constituer une piste intéressante et innovante dans le cadre d'un accompagnement des mères, dans la période de profonds changements qui fait suite à la naissance d'un enfant. D'une part, le récit de vie participe au 
réaménagement de l'identité narrative, portion de l'identité d'une personne, et, en facilitant la réorganisation identitaire des mères, contribue à leur santé. D'autre part, le carnet de bord se révèle un intermédiaire pertinent entre les femmes et la sage-femme. Par ailleurs, l'introduction du carnet de bord dans un dispositif d'éducation pour la santé en périnatalité sous-tend la posture pédagogique de la sage-femme éducatrice. L'accompagnement éducatif des mères vise alors le développement de leurs potentialités et retrouve la dimension d'un échange. Il est beaucoup plus qu'une simple transmission de connaissances ou d'informations et ne peut être comparé à une prise en charge médicale dissymétrique. Comme l'a montré notre étude, les femmes sont acteurs du processus et l'engagement de part et d'autre est indispensable à la poursuite de l'entreprise. Ceci peut être perçu comme une difficulté mais c'est aussi ce qui fait tout l'intérêt d'une telle démarche qui pourrait être proposée dès la période prénatale

\section{Contributions}

Marie-Reine Bernard et Chantal Eymard sont les promoteurs de l'étude. Marie-Reine Bernard a mené les entretiens, réalisé l'analyse de contenu et rédigé l'article. Chantal Eymard a supervisé l'analyse de contenu et révisé l'article

\section{Références}

1. Golse B. Paradoxes et limites de la prévention précoce. In : Neyrand G et al. (dir.) Famille et petite enfance. Mutation des savoirs et des pratiques. Ramonville StAgne : Eres, 2006:239-55.

2. Glatigny-Dallay E, Sutter-Dallay AL, Loustau N. Une approche pluridisciplinaire des troubles psychiatriques de la période périnatale. Le journal des psychologues 2008;261:26-33.

3. Neyrand G. La dynamique d'un réseau de prévention psychique. Résistances et perspectives. RFAS 2004;1:10325 .

4. Dugnat M. Renouveler la prévention. In : Neyrand G et al. (dir.). Famille et petite enfance. Mutation des savoirs et des pratiques. Ramonville St-Agne : Eres, 2006 : 257-74.
5. Soliday E, Garofalo JP, Rogers D. Expressive writing intervention for adolescents' somatic sympotms and mood. J Clin Child Adolesc Psychol 2004;33:792-801.

6. Mastel-Smith BA, McFarlane J, Sierpina M, Malecha A, Haile B. Improwing depressive symptoms in community-dwelling older adults: a psychosocial intervention using life review and writing. J Gerontol Nurs 2007;33:13-9.

7. Gellaitry G, Peters K, Bloomfield D, Horne R. Narrowing the gap: the effects of an expressive writing intervention on perceptions of actual and ideal emotional support in women who have completed treatment for early stage breast cancer. Psychooncology 2010;19:77-84.

8. Niewiadomski C. Education pour la santé, appartenance de groupe et émergence d'une identité singulière. L'exemple de l'histoire de vie en alcoologie. Les sciences de l'éducation en question, Éduquer à ou pour la santé ? Quels enjeux pour la formation et pour la recherche ? Questions vives 2004;5:113-41.

9. Danon-Boileau L. Narrativité, conscience de soi et accès à la culture chez l'enfant. In : Golse B, Missonnier S. Récit, attachement et psychanalyse. Pour une clinique de la narrativité. Ramonville Saint-Agne : Édition Erès, 2005:21-8.

10. Eymard C. Essai de modélisation entre éducation et santé. Les sciences de l'éducation en question, Éduquer à ou pour la santé ? Quels enjeux pour la formation et pour la recherche ? Questions vives 2004;5:13-33.

11. World Health Organization. Skills for health. Geneva 2003 [On-line] Disponible sur : http://whqlibdoc.who. int/publications/2003/924159103X.pdf

12. HAS-INPES. Guide méthodologique. Structuration d'un programme d'éducation thérapeutique du patient dans le champ des maladies chroniques, 2007 [On-line] Disponible sur : http://www .has-sante. fr

13. Lecorps P, Paturet JB. Santé publique : du biopouvoir à la démocratie. Rennes : ENSP, 1999.

14. Mercier M, Schraub S. Qualité de vie : quels outils de mesure ? $27^{\text {es }}$ journées de la SFSPM, Deauville novembre 2005 .

15. Ferron C. Education pour la santé. In : Bourdillon F (dir.). Agences régionales de santé. Promotion, prévention et programmes de santé. INPES éditions, 2010 : 144-48 [On line] Disponible sur : www . inpes . sante. fr

16. Eymard C. Des modèles de l'éducation et de la santé à l'activité d'éducation thérapeutique. In : Foucaud J \& al. Éducation thérapeutique du patient. Modèles, pratique et évaluation. INPES, septembre 2010:39-52 [On line] Disponible sur: www . inpes. sante. fr

17. BDSP- Glossaire Européen en santé publique. [On line] Disponible sur : http://www.bdsp.ehesp.fr/ 
18. Haute autorité de santé. Recommandations pour la pratique clinique. Préparation à la naissance et à la parentalité (PNP), 2005 [On line] Disponible sur : http://www. has-sante.fr

19. IPCEM. Questions à propos de l'éducation thérapeutique, juillet 2009 [On line] Disponible sur : http://www. ipcem.org

20. Rajon AM. Survivre à l'arrivée d'un enfant. L'école des parents 2007;563 HS:36-8.

21. Stern D. La constellation maternelle. Paris : CalmannLévy, 1997.

22. Revault d'Allones C. Etre, faire, avoir un enfant. Paris : Payot et Rivages, 2004.

23. Bergeret-Amselek C. Le mystère des mères. Paris : Le grand livre du mois, 1999.

24. Ricœur P. Soi-même comme un autre. Paris : Seuil, 1990.

25. Kaufmann JC. L'invention de soi. Une théorie de l'identité. Paris : Hachette littératures, Coll. Armand Colin, 2004.

26. Golse B, Missonnier S. Récit, attachement et psychanalyse. Pour une clinique de la narrativité. Ramonville SaintAgne : Édition Erès, 2005.

27. Abels C. L'histoire de vie comme outil d'historicité. Revue Histoire de vie. Rennes : Presses Universitaires de Rennes 2002;3:17-20.

28. Pineau G, Legrand JL. Les histoires de vie. Paris : Presses Universitaires de France, 2007.

29. Bardin L. L'analyse de contenu. Paris : Presses Universitaires de France, 2007.

30. Bydlowski M. La dette de vie. Itinéraire psychanalytique de la maternité. Paris : Presse Universitaires de France, 2005.
31. Winnicott DW. De la pédiatrie à la psychanalyse. Paris : Payot, 1969.

32. Ryan R, Larguardia J. Buts personnels, besoins psychologiques fondamentaux et bien-être : Théorie de l'autodétermination et applications. Revue québécoise de psychologie 2000;21:281-302.

33. Buber M. Je et Tu. Paris : Aubier, 1969.

34. Ricoeur P. Les paradoxes de l'identité. L'information psychiatrique 1996;3:201-6.

35. Dumas D. Sans père et sans parole. La place du père dans l'équilibre de l'enfant. Paris : Hachette littératures, 1999.

36. Ardoino J. L'éducation en tant qu'altération des personnes, ou la recherche prenant le changement pour objet, Québec 1996 [On line] Disponible sur : http:// jardoino.club.fr/TextesThematique.htm

37. Benvéniste E. Problèmes de linguistique générale, 1. Paris : Gallimard, 1966.

38. Billotte G. L'histoire de vie, un travail de recomposition de soi. In : Filloux JC. (Coord.) Analyse d'un récit de vie. Paris : Presses Universitaires de France, 2005:87-97.

39. Bruner J. Pourquoi racontons-nous des histoires ? Le récit au fondement de la culture et de l'identité individuelle. Paris : Pocket Agora, 2005.

Correspondance et offprints : Marie-Reine Bernard. Département Sciences de l'éducation, Aix Marseille I-Université de Provence, Unité mixte de recherche «Apprentissage Didactique Éducation Formation », EU3M (École Universitaire de Maïeutique Marseille Méditerranée), Campus Santé Nord, Boulevard Pierre Dramard, 13344 Marseille Cedex 15, France

Mailto : Marie-reine.bernard@univmed.fr 